\title{
Selectively increased trunk mobility with FITS therapy: a preparatory stage for correction of idiopathic scoliosis Marianna Białek*1, Wiesław Chwała ${ }^{2}$ and Tomasz Kotwicki ${ }^{3}$
}

Address: ${ }^{1}$ FITS Center Kielce, Poland, ${ }^{2}$ Department of Biomechanics, Laboratory of Biokinetics, Academy of Physical Education, Cracow, Poland and ${ }^{3}$ Department of Pediatric Orthopedics and Traumatology University of Medical Sciences, Poznan, Poland

* Corresponding author

from $5^{\text {th }}$ International Conference on Conservative Management of Spinal Deformities

Athens, Greece. 3-5 April 2008

Published: 15 January 2009

Scoliosis 2009, 4(Suppl I):O29 doi:I0.II86/I748-7|6I-4-SI-O29

This abstract is available from: http://www.scoliosisjournal.com/content/4/SI/O29

(C) 2009 Białek et al; licensee BioMed Central Ltd.

\section{Background}

Reduced spine mobility inside the scoliotic curve can be an obstacle for effective correction of idiopathic scoliosis with physiotherapy or bracing.

\section{Purpose}

To assess the effect of a session of specific FITS physiotherapy on trunk mobility.

\section{Methods}

Thirteen girls aged $14.7+/-1.4$ years, presenting single thoraco-lumbar scoliosis of $28.6+/-7.5$ degrees of the Cobb angle, were examined. They presented coronal imbalance comprising a protruding concave hip, a deeper concave waist and the medial occipital plumb line shift of $1.5+/-0.5 \mathrm{~cm}$ to the convexity.

Trunk mobility was assessed before and after one session of FITS physiotherapy, by measuring the range of: (1) frontal trunk shift towards concavity, (2) lateral trunk inclination towards convexity, (3) trunk rotation towards correction, (4) thoracic spine length in forward flexion, (5) lumbar spine length in forward flexion. The control group consisted of the same patients who were examined once again one month later. They underwent identical measurements before and after one session of an unspecific body massage.

\section{Results}

All the parameters apart the thoracic spine flexion revealed significant increase of the range of the tested movements $(\mathrm{p}<0.05)$. After one FITS session, the trunk shift, expressed as the percentage of the trunk height, increased of $3.9 \pm 2.1 \%$, from $6.7 \pm 2.3 \%$, to $10.6 \pm 2.5 \%$ $(\mathrm{p}<0.0001)$. However, after unspecific massage, the trunk shift increased but not significantly.

\section{Conclusion}

A specific FITS session increased trunk mobility towards the correction of scoliosis more than unspecific massage. This can increase the effectiveness of subsequent corrective patterns of movement.

\section{References}

I. Suzuki N, Inami T, Ono T, Kohno K, Asher MA: Analysis of Posterior Trunk Symmetry Index (POTSI) in Scoliosis. Stud Health Technol Inform 1999, 59:8I-84.

2. Bialek M, M'hango A: FITS concept: Functional Individual Therapy of Scoliosis. The Conservative Scoliosis Treatment. Stud Health Technol Inform 2008, 135:250-261. 